\title{
Conocimiento del riesgo de presentar un infarto de miocardio y las barreras para el acceso al estilo de vida saludable
}

\author{
Clara Saldarriaga ${ }^{a, b, c, *}$, Lennis Bedoya ${ }^{d}$, Laura Gómez ${ }^{d}$, Laura Hurtado ${ }^{d}$, \\ Juliana Mejía ${ }^{e}$ y Natalia González ${ }^{e}$
}

a Clínica CardioVID, Medellín, Colombia

b Sección de Cardiología, Universidad de Antioquia, Medellín, Colombia

c Programa de Cardiología, Universidad Pontificia Bolivariana, Medellín, Colombia

d Medicina, Universidad Pontificia Bolivariana, Medellín, Colombia

e Oficina de Apoyo a la Investigación Clínica CardioVID, Medellín, Colombia

Recibido el 26 de marzo de 2015; aceptado el 14 de julio de 2015

Disponible en Internet el 28 de agosto de 2015

\section{PALABRAS CLAVE \\ Factores de riesgo; \\ Estilo de vida; \\ Enfermedades \\ cardiovasculares}

\begin{abstract}
Resumen
Introducción: La falta de infomación sobre los factores de riesgo para presentar un infarto agudo de miocardio es un elemento que permite incrementar su prevalencia en nuestra comunidad. Objetivos: Identificar el conocimiento de los factores de riesgo cardiovasculares y las barreras para su modificación en una población de Medellín, Colombia.

Metodología: Estudio descriptivo de corte transversal, se incluyó a la población que asistió a las jornadas del día mundial del corazón y se registraron variables antropométricas. Adicionalmente se indagó sobre el conocimiento de la principal causa de muerte en Colombia y en el mundo, su intención de realizar cambios en el estilo de vida y las barreras para implementarlo. Se realizó análisis bivariado según el sexo.

Resultados: Se incluyeron 1.324 adultos. El $46 \%$ de los hombres vs. el $41,4 \%$ de las mujeres identificó la enfermedad coronaria como la primera causa de muerte. La mayoría de los hombres y las mujeres manifestaron su interés en iniciar un estilo de vida saludable $(91,8$ vs. $94,5 \%$ $\mathrm{p}=0,07)$. La falta de tiempo fue la principal barrera. El $2,2 \%$ de los hombres y $2,6 \%$ de las mujeres manifestaron desconocer cómo pueden iniciar un estilo de vida saludable.

Conclusión: Existe un pobre reconocimiento de la enfermedad coronaria como causa de muerte, especialmente en las mujeres, la falta de tiempo y los factores económicos son la principal barrera para implementar cambios en los factores de riesgo modificables.

(c) 2015 Sociedad Colombiana de Cardiología y Cirugía Cardiovascular. Publicado por Elsevier España, S.L.U. Este es un artículo Open Access bajo la CC BY-NC-ND licencia (http:// creativecommons.org/licencias/by-nc-nd/4.0/).
\end{abstract}

\footnotetext{
* Autor para correspondencia.

Correo electrónico: clarais@une.net.co (C. Saldarriaga).
} 


\section{KEYWORDS}

Risk factors;

Life style;

Cardiovascular

diseases

\section{Knowledge of the risk of a myocardial infarction presentation and barriers to a healthy life style in a population of Medellin, Colombia}

\begin{abstract}
Introduction: The lack of information about cardiovascular risk factors is an element that increases their prevalence in our community.

Objective: To identify the knowledge of cardiovascular risk factors and barriers for their modification in a population of men and women of Medellin, Colombia.

Methodology: A descriptive, cross-sectional study was conducted including the population that assisted to the world heart day event. Anthropometric variables, vital signs and blood glucometry were performed. People were asked about their knowledge of the main cause of dead in Colombia and worldwide, their intention to implement changes in their lifestyle and the barriers to implement it. A bivariate analysis was performed by sex.

Results: 1,324 adults were included; 46 vs. $41.4 \%$ of men and women identified coronary heart disease as the first cause of dead worldwide and in Colombia. The majority of men and women talk about their interest in changing their life style $(91.8 \mathrm{vs} .94 .5 \% \mathrm{p}=.07)$. Lack of time was identified as the leading barrier to access a healthy lifestyle. $2.2 \%$ of men and $2.6 \%$ of women didn't know how to start a healthy lifestyle.

Conclusion: There is a poor recognition of coronary heart disease as a cause of dead, especially in women; lack of time and economical factors are the main barrier to implement changes in modifiable risk factors.

() 2015 Sociedad Colombiana de Cardiología y Cirugía Cardiovascular. Published by Elsevier España, S.L.U. This is an open access article under the CC BY-NC-ND license (http:// creativecommons.org/licenses/by-nc-nd/4.0/).
\end{abstract}

\section{Introducción}

El infarto de miocardio es la principal causa de mortalidad en el mundo, superando a enfermedades como el cáncer e incluso a la violencia en los países en vía de desarrollo ${ }^{1}$, adicionalmente se estima que con el incremento observado en la expectativa de vida se aumentará la incidencia de nuevos casos de síndrome coronario agudo ${ }^{2}$ y por esta razón la promoción de los estilos de vida saludable y la educación a la comunidad sobre el conocimiento y la modificación de los factores de riesgo cardiovasculares debe convertirse en una prioridad y en una política de salud en Colombia. Las estadísticas de la Asociación Americana del Corazón han reportado que durante la última década se ha presentado una disminución en la aparición de nuevos casos de infarto en la población masculina, no obstante, no ha sido posible disminuir la incidencia de la enfermedad coronaria en la población femenina ${ }^{3,4}$, la desinformación y las barreras para acceder a un estilo de vida saludable se han planteado como dos posibles explicaciones a este comportamiento diferente entre sexos respecto a la morbilidad cardiovascular y por esta razón el presente estudio tiene como objetivo identificar el conocimiento de los factores de riesgo cardiovasculares y las barreras para su modificación en una población de mujeres y hombres de la ciudad de Medellín.

\section{Metodología}

Se realizó un estudio descriptivo de corte transversal, que incluyó a la población de hombres y mujeres, mayores de 18 años de edad, que asistieron de forma voluntaria a las jornadas del día mundial del corazón durante los años 2012 y 2013 en varios centros comerciales de la ciudad de Medellín. Se diseñó una encuesta de 8 preguntas con respuestas de selección múltiple que debía ser contestada por las personas, sin ayuda de los encuestadores en la cual se indagó sobre el conocimiento de la principal causa de muerte en Colombia y en el mundo, su intención de realizar cambios en el estilo de vida, las barreras que no les permiten implementarlos y el antecedente de la hipertensión, la diabetes, la dislipidemia y el tabaquismo. Adicionalmente, durante una de las dos jornadas se registraron los signos vitales y el índice de masa corporal. Esta información fue recolectada en una base de datos de Excel y doblemente verificada.

Para el análisis univariado se resumieron las variables cualitativas como frecuencias absolutas y relativas, para las cuantitativas como media y desviación estándar como medida de dispersión dada la ausencia de valores extremos. Posteriormente se realizó una análisis bivariado según el sexo para lo cual se calcularon los valores de $\mathrm{p}$ mediante Chi cuadrado en el caso de variables cualitativas. Para el procesamiento de los datos se realizó en el programa SPSS V.20, en todos los casos se consideró como diferencia estadísticamente significativa los que presentaran valores de $p$ menores de 0,05.

\section{Resultados}

Se incluyeron 1.324 pacientes, con edad promedio de $48,8 \pm 17,1$ años, el $30,5 \%(n=404)$, eran de sexo masculino y $69,5 \%$ era de sexo femenino $(n=920)$. Se realizó el registro de los signos vitales, la talla y el peso durante la primera jornada y estos datos estuvieron disponibles en el $36,9 \%$ de 
Tabla 1 Identificación de la principal causa de muerte en el mundo

\begin{tabular}{lccl}
\hline $\begin{array}{l}\text { Causa de } \\
\text { muerte en el } \\
\text { mundo }\end{array}$ & $\begin{array}{l}\text { Hombres } \\
\mathrm{n}=404\end{array}$ & $\begin{array}{l}\text { Mujeres } \\
\mathrm{n}=920\end{array}$ & $\begin{array}{l}\text { Valor } \\
\text { de } \mathrm{p}\end{array}$ \\
\hline $\begin{array}{l}\text { Accidentes } \\
\text { Cáncer }\end{array}$ & $31(7,7 \%)$ & $76(8,3 \%)$ & 0,024 \\
Infarto & $186(19,8 \%)$ & $252(27,4 \%)$ & \\
Violencia & $107(26,5 \%)$ & $381(41,4 \%)$ & \\
\hline
\end{tabular}

Tabla 2 Identificación de la principal causa de muerte en Colombia

\begin{tabular}{lccc}
\hline $\begin{array}{l}\text { Causa de } \\
\text { muerte en } \\
\text { Colombia }\end{array}$ & $\begin{array}{l}\text { Hombres } \\
\mathrm{n}=404\end{array}$ & $\begin{array}{l}\text { Mujeres } \\
\mathrm{n}=920\end{array}$ & $\begin{array}{l}\text { Valor } \\
\text { de } \mathrm{p}\end{array}$ \\
\hline Accidentes & $35(8,7)$ & $66(7,2)$ & 0,694 \\
Cáncer & $71(17,6)$ & $180(19,6)$ & \\
Infarto & $157(39)$ & $364(39,5)$ & \\
Violencia & $139(34,4)$ & $310(33,7)$ & \\
\hline
\end{tabular}

Tabla 3 Intención de realizar cambios en el estilo de vida

\begin{tabular}{lccl}
\hline $\begin{array}{l}\text { Interés en } \\
\text { realizar } \\
\text { cambios }\end{array}$ & $\begin{array}{l}\text { Hombres } \\
\mathrm{n}=404\end{array}$ & $\begin{array}{l}\text { Mujeres } \\
\mathrm{n}=920\end{array}$ & $\begin{array}{l}\text { Valor } \\
\text { de } \mathrm{p}\end{array}$ \\
\hline Sí & $371(91,8 \%)$ & $869(94,5 \%)$ & 0,071 \\
No & $32(8,2 \%)$ & $51(5,5 \%)$ & \\
\hline
\end{tabular}

la población encuestada. Para este grupo de pacientes se encontró que el promedio de índice de masa corporal fue $26,7 \pm 11,2$, la presión arterial sistólica y diastólica fueron $116 \pm 17,4$ y $72 \pm 9$, respectivamente.

Los factores de riesgo cardiovasculares más relevantes reportados en la encuesta fueron el tabaquismo activo $(8,8 \%)$, la dislipidemia $(21,7 \%)$, la diabetes $(17,6 \%)$ y la hipertensión arterial $(60,4 \%)$, de los individuos encuestados.

La minoría de la población tenía antecedente de la enfermedad coronaria $(4,7 \%)$, por lo cual se concluye que esta era una muestra de personas «sanas» en su mayoría y constituyen un escenario de prevención primaria.

Respecto al objetivo principal del estudio, se logró determinar que el 46 vs. $41,4 \%$ de los hombres y las mujeres identificó de forma correcta a la enfermedad coronaria como la primera causa de muerte en el mundo, encontrándose diferencias estadísticamente significativas por sexo (tabla 1), ambas poblaciones a su vez identificaron con mayor frecuencia a la enfermedad coronaria como causa principal de la muerte en Colombia (39 vs. 39,5\%), en segundo lugar el cáncer, en tercer lugar la violencia y por último los accidentes como causas importantes de mortalidad (tabla 2). La mayoría de los hombres y las mujeres manifestaron su interés en iniciar un estilo de vida saludable $(91,8$ vs. $94,5 \% p=0,07)$ (tabla 3$)$. La población masculina identificó de manera significativa el inicio de la actividad física como el principal cambio que estarían dispuestos a realizar (53 vs. 46,2\%), en segundo lugar la disminución del
Tabla 4 Cambios que realizaría en el estilo de vida

\begin{tabular}{lccl}
\hline Cambios & $\begin{array}{l}\text { Hombres } \\
\mathrm{n}=404\end{array}$ & $\begin{array}{l}\text { Mujeres } \\
\mathrm{n}=920\end{array}$ & $\begin{array}{l}\text { Valor } \\
\text { de } \mathrm{p}\end{array}$ \\
\hline Bajar de peso & $85(21 \%)$ & $227(24,7 \%)$ & 0,014 \\
Dieta & $70(17,3 \%)$ & $205(22,3 \%)$ & \\
Dejar de fumar & $26(6,4 \%)$ & $38(4,1 \%)$ & \\
Ejercicio & $214(53 \%)$ & $425(46,2 \%)$ & \\
\hline
\end{tabular}

Tabla 5 Barreras para acceder a un estilo de vida saludable

\begin{tabular}{|c|c|c|c|}
\hline Barrera & $\begin{array}{l}\text { Hombres } \\
n=404\end{array}$ & $\begin{array}{l}\text { Mujeres } \\
n=920\end{array}$ & $\begin{array}{l}\text { Valor } \\
\text { de } p\end{array}$ \\
\hline No le interesa & $106(26,2 \%)$ & $241(26,2 \%)$ & $<0,005$ \\
\hline Falta de tiempo & $193(47,8 \%)$ & $319(34,7 \%)$ & \\
\hline $\begin{array}{l}\text { Limitaciones } \\
\text { económicas }\end{array}$ & $20(5 \%)$ & $76(8,3 \%)$ & \\
\hline Desconocimiento & $9(2,2 \%)$ & $24(2,6 \%)$ & \\
\hline
\end{tabular}

peso ( 21 vs. $24,7 \%$ ) y en tercer lugar la modificación en la dieta (17,3 vs. $22,3 \%$ ) (tabla 4$)$. La falta de tiempo fue identificada como la principal barrera de acceso al estilo de vida saludable. Se encontró un $26 \%$ de la población a la cual no le interesa llevar un estilo de vida saludable. Adicionalmente, las dificultades económicas se identificaron como una barrera para la implementación del estilo de vida saludable y el desconocimiento en el 2,2\% de los hombres y $2,6 \%$ de las mujeres que manifestaron no saber cómo modificar su riesgo de presentar un infarto (tabla 5).

\section{Discusión}

Los resultados del presente estudio identificaron que en la población evaluada existe un gran desconocimiento respecto a la importancia de la enfermedad coronaria como la principal causa de mortalidad en Colombia y el mundo, pues menos del $50 \%$ de los hombres y las mujeres la identificaron de forma correcta. Esta información es de gran relevancia porque resalta la importancia de diseñar estrategias educativas dirigidas a la población general que permitan crear consciencia sobre la importancia de controlar los factores de riesgo modificables. El análisis por sexos mostró que existen diferencias significativas respecto al conocimiento de las causas de mortalidad en el mundo, pues las mujeres encuestadas identificaron en un menor porcentaje a la enfermedad coronaria como causa de muerte. Existen reportes similares en la literatura e incluso se plantea a la desinformación como una de las causas de la alta prevalencia de enfermedad cardiovascular en las mujeres ${ }^{5}$. En el año 2003 la Asociación Americana del Corazón realizó una encuesta en los Estados Unidos donde se demostró que el 35\% de las mujeres identificaban el cáncer de mama como la principal causa de muerte, mientras que solo el $13 \%$ consideraban a la enfermedad coronaria como una enfermedad importante y mortal ${ }^{6,7}$. Desde entonces se creó la campaña del «vestido rojo» que ha permitido mejorar el conocimiento de las mujeres sobre la importancia de esta enfermedad ${ }^{8}$. No obstante, es llamativa la diferencia en las campañas que existen para la 
prevención del cáncer de mama vs. las campañas para la prevención de la enfermedad coronaria, las primeras han sido muy exitosas, han logrado una gran conscientización de la población femenina y la movilización de muchos recursos de la industria privada, probablemente es tiempo de replantear nuestras prioridades en prevención de las enfermedades y hacer un mayor énfasis en aquellas de mayor prevalencia e impacto clínico.

Es importante resaltar que la población incluida en el estudio estaba conformada por adultos de la ciudad de Medellín, en edad media de la vida y con un bajo índice de comorbilidad, lo cual puede generar un sesgo de selección. Adicionalmente, la mayoría eran mujeres en la quinta década de la vida, este grupo etáreo es un blanco muy importante desde el punto de vista de la prevención cardiovascular pues representa los años iniciales de la menopausia, en los cuales se presentan grandes cambios como el incremento del tejido adiposo abdominal, la disminución del colesterol HDL y el aumento del colesterol LDL en relación a la supresión de la producción ovárica de los estrógenos los cuales son fenómenos que disparan la formación e inestabilización de placas aterogénicas ${ }^{9-12}$.

Generalmente, se encontró una gran motivación para iniciar actividades encaminas a llevar un estilo de vida saludable y las prioridades encontradas fueron la disminución del peso y los cambios en la alimentación principalmente en las mujeres mientras que los hombres consideraron con mayor frecuencia volverse físicamente activos y dejar de fumar. Esta iniciativa debe apoyarse con campañas de trabajo con la comunidad utilizando metodologías que han demostrado ser exitosas en el mundo, como aquellas en las cuales se incluyan fases de educación, acompañamiento durante la implementación de los cambios del estilo de vida y finalmente se evalúe el impacto de la intervención en el riesgo cardiovascular ${ }^{13-16}$.

Las diferencias significativas encontradas en las barreras para acceder a un estilo de vida saludable fueron la falta de tiempo en los hombres y las limitaciones económicas en las mujeres, estos resultados son similares a los que reporta la literatura mundial, donde además se resalta el bajo nivel educativo como una barrera para acceder al cuidado de la salud $^{17-20}$

A la luz de los resultados del presente estudio es indispensable iniciar en Colombia campañas que informen a la comunidad general respecto a la importancia de la enfermedad coronaria como causa de la morbilidad y la mortalidad, educar desde la infancia para implementar la actividad física y la alimentación saludable desde temprana edad como respuesta a la prevalencia de las enfermedades cardiovasculares en los países en vía de desarrollo, pues los países latinoamericanos han experimentado grandes cambios en la última década, con un incremento en la expectativa de vida que se traduce en un aumento en la morbimortalidad generada por la enfermedad cardiovascular que es potencialmente prevenible y que en la actualidad genera grandes costos al sistema de salud.

Las limitaciones del presente estudio es que las personas que llenaron la encuesta son de una población urbana que no representa completamente a la población colombiana, es posible que la población rural presente un desconocimiento mayor y es necesario realizar un estudio a mayor escala que permita evaluar estos aspectos en todo el país.

\section{Conclusiones}

El presente estudio muestra que en una población urbana de la ciudad de Medellín existe un pobre reconocimiento de la enfermedad coronaria como causa de muerte en Colombia y en el mundo. Aunque existe una motivación importante para realizar cambios en el estilo de vida, existen barreras para implementarlo como la falta de tiempo y los factores económicos.

\section{Responsabilidades éticas}

Protección de personas y animales. Los autores declaran que para esta investigación no se han realizado experimentos en seres humanos ni en animales.

Confidencialidad de los datos. Los autores declaran que han seguido los protocolos de su centro de trabajo sobre la publicación de datos de pacientes.

Derecho a la privacidad y consentimiento informado. Los autores han obtenido el consentimiento informado de los pacientes y/o sujetos referidos en el artículo. Este documento obra en poder del autor de correspondencia.

\section{Conflicto de intereses}

Los autores declaran no tener ningún conflicto de intereses.

\section{Agradecimientos}

Los autores agradecen al doctor Juan Carlos González, director científico de la clínica CardioVID su colaboración para el proceso de recolección de la información que permitió realizar este estudio.

Fuentes de apoyo: el estudio se realizó gracias al apoyo de la clínica CardioVID.

\section{Bibliografía}

1. Mozaffarian D, Benjamin EJ, Go AS, Arnett DK, Blaha MJ, Cushman M, et al., American Heart Association Statistics Committee and Stroke Statistics Subcommittee. Heart disease and stroke statistics-2015 update: a report from the American Heart Association. Circulation. 2015;131(4):e29-322.

2. Huffman MD. Cardiovascular health in low- and middle-income countries. Curr Probl Cardiol. 2014;39(11):399-419.

3. Shaw LJ, Lewis JF, Hlatky MA, Hsueh WA, Kelsey SF, Klein R, et al. Women's ischemic syndrome evaluation: current status and future research directions: report of the National Heart Lung and Blood Institute workshop: October 2-4, 2002: Section 5: gender-related risk factors for ischemic heart disease. Circulation. 2004;109(6):e56-8.

4. Shaw LJ, Bairey Merz CN, Pepine CJ, Reis SE, Bittner V, Kelsey SF, et al. Insights from the NHLBI-Sponsored Women's Ischemia Syndrome Evaluation (WISE) Study: Part I: gender differences in traditional and novel risk factors, symptom evaluation, and gender-optimized diagnostic strategies. J Am Coll Cardiol. 2006;47 3Suppl:S4-20.

5. Mosca L, Ferris A, Fabunmi R, Robertson RM, American Heart Association. Tracking women's awareness of heart disease: 
an American Heart Association national study. Circulation. 2004;109(5):573-9.

6. McSweeney JC, Cody M, Crane PB. Do you know them when you see them? Women's prodromal and acute symptoms of myocardial infarction. J Cardiovasc Nurs. 2001;(3):26-38.

7. Galick A, D’Arrigo-Patrick E, Knudson-Martin C. Can anyone hear me? Does anyone see me? A qualitative meta-analysis of women's experiences of heart disease. Qual Health Res. 2015, pii: 1049732315584743. [Publicación electrónica] PubMed PMID: 25924615.

8. Long T, Taubenheim A, Wayman J, Temple S, Ruoff B. The heart truth:" using the power of branding and social marketing to increase awareness of heart disease in women. Soc Mar Q. 2008;14(3):3-29.

9. Pepine CJ, Kerensky RA, Lambert CR, Smith KM, von Mering GO, Sopko G, et al. Some thoughts on the vasculopathy of women with ischemic heart disease. J Am Coll Cardiol. 2006;47 3 Suppl:S30-5.

10. Nohria A, Vaccarino V, Krumholz HM. Gender differences in mortality after myocardial infarction. Why women fare worse than men. Cardiol Clin. 1998;16(1):45-57.

11. Stangl V, Baumann $G$, Stangl K. Coronary atherogenic risk factors in women. Eur Heart J. 2002;23(22):1738-52.

12. Kuulasmaa K, Tunstall-Pedoe H, Dobson A, Fortmann S, Sans S, Tolonen $\mathrm{H}$, et al. Estimation of contribution of changes in classic risk factors to trends incoronary-event rates across the WHO MONICA Project populations. Lancet. 2000;355(9205):675-87.

13. Krummel DA, Koffman DM, Bronner Y, Davis J, Greenlund $\mathrm{K}$, Tessaro I, et al. Cardiovascular health interventions in women: What works? J Womens Health Gend Based Med. 2001;10(2):117-36.

14. Jane M, Foster J, Hagger M, Pal S. Using new technologies to promote weight management: a randomised controlled trial study protocol. BMC Public Health. 2015;15:509.

15. Yanek LR, Becker DM, Moy TF, Gittelsohn J, Koffman DM. Project Joy: faith based cardiovascular health promotion for African American women. Public Health Rep. 2001;116 Suppl 1:68-81.

16. Sallis R, Franklin B, Joy L, Ross R, Sabgir D, Stone J. Strategies for promoting physical activity in clinical practice. Prog Cardiovasc Dis. 2015;57(4):375-86.

17. Trinh-Shevrin C, Islam NS, Nadkarni S, Park R, Kwon SC. Defining an integrative approach for health promotion and disease prevention: a population health equity framework. J Health Care Poor Underserved. 2015;26 2 Suppl:146-63.

18. Liu J, Davidson E, Bhopal R, White M, Johnson M, Netto G, et al. Adapting health promotion interventions to meet the needs of ethnic minority groups: mixed-methods evidence synthesis. Health Technol Assess. 2012;16(44):1-469.

19. McGuire AM, Anderson DJ, Fulbrook P. Perceived barriers to healthy lifestyle activities in midlife and older Australian women with type 2 diabetes. Collegian. 2014;21(4):301-10.

20. Liao Y, Tucker P, Okoro CA, Giles WH, Mokdad AH, Harris VB, Division of Adult and Community Health, National Center for Chronic Disease Prevention and Health Promotion, Centers for Disease Control and Prevention (CDC). REACH 2010 Surveillance for Health Status in Minority Communities - United States, 2001-2002. MMWR Surveill Summ. 2004;53(6):1-36. 